\title{
Contents, Vol. 10, 1903
}

Jnhalts -Verzeichnis.

Opiginalapbeiten.

Alexander, L., Ein weiterer Fall von in den Glaskörper

vordringender Arterienschlinge 188

Baas, J. H, Bindeliautentzündung infolge von Ansteckungdurch Sekret des „,infektiösen

Scheidenkatarrhs" beieiner Kuh 49

- $\quad$ Ein Fall von symmetrischen Geschwülsten der Thränen-

drüsen, der Lider, von Mund-Schleimhaut-Drüsen . 184 Baas, K., Primäres Endotheliom der

Cornea (Hierzu Fig. 1

auf Tafel VI) . . 485

Cohn, Paul, Totales Ankyloblepharon durch Pemphigus

mit Ausgang in völlige Heilung 421

-Gräflin, Arthur, Experimentelle Untersuchungen über den

schädlichen Einfluss von pulverförmigen Anilinfarben

auf die Schleimhaut des Kaninchenauges 193

Gutmann, Adolf, Ueber kollagenes und protoplasmatisches

Grewebe der menschlichen Iris (Hierzu Taf. I) . 8

Hadano, Beitrag zur Kenntnis der Keratitis disciformis

(Hierzu Fig. 2 auf Taf. VI) 500

Hála, Adolf, Ein seltenes Symptom der Augensyphilis bei

Neugeborenen 85

Halben, R., Ein Fall geheilter Wortblindheit mit Persistenz

rechtsseitiger Hemianopsie 406, 487

Hanke, Victor, Ein bi§her unbekannter Bacillus, der Er-

reger des typischen Ringabscesses der Cornea . . 373 Koerber, H., Bericht über ein Papillom der Conjunctiva

sclerae und ein Lymphom der Plica semilunaris (Hierzu

Fig. 1 auf Tafel V) 146

- $\quad$ Ueber die Beziehungen zwischen Iriskolobom und

Korektopie (Hierzu Fig. 2 auf Taf. V) . . . ... 149

$-\mathrm{IY}-$

Krauss, W., Uebei· Jequiritol (Hierzu Taf. II-IV) . . 34, ï22

Krüger, A. E., Die bei Erkrankungen. des Schläfenlappensund des Stirnlappens beobachteten Symptome, mit be-sonderer Berücksichtigung der okularen Symptome.I.: Erkrankungen des

Schläfenlappens $\quad 505$

Kuhut, H., Zur ïechnik der Irisausschneidungen . . . 219

Kurzezunge und Pollack, B., Ein Fall von primärer Neu-

bildung auf der Papule des Opticus 302

Laqueur, Beitrag zur Lehre von den hereditären Erkrankungen des Auges 477 
Lohnstein, Rudolf, Eine einfache Vorrichtung zur

Refraktionsbestimmung im umgekehrten Bilde ... 1

Michel, v., Ueber einseitige familiäre und angeborene

Innervationsstörungen des Halssympathicus . . . 18.1

Mörehen, Friedrich, Die Erscheinungen bei Erkrankungendes Sehhügels, mit spezieller

Berücksichtigung derokularen Symptome 272

- Die Erscheinungen bei Erkrankungen des Hirnschenkels,

mit besonderer Berücksichtigung der okularenSymptome 382

Pagenstecher, Ad. EL, Ueber Staroperationen mit besonderer Berücksichtigung der

Nachstaroperationen . 206

Pfalz, Die Netzhautablösung als Unfallfolge 261

Pollack, B., s. Kurzezunge u. P.

Sicller-Huguenin, llerstellungsweise der Jodoformstäbchen

und -plättchen für die intraokuläre Desinfektion . 108

Wachtler, G-uido, Zur Frage der in den Glaskörper vor-

dringenden Arterienschlingen 425

Weiss, Edwart, Pathologisch-anatomischer Beitrag zur

Frage der Orbitalphlegmone 16,91

Bericht über die deutsehe ophthalmolog .isehe Littepatur.

Anatomie des Auges. Von Prof. Dr. Sobotta in Würz-

burg. (II. Semester 1902) 50

Üntersuchungsmethoden. Von Prof. Dr. Dimmer in $(3 / 8 a z$.

(1902) 152

Angeborene Anomalien. Von Priv.-Doc. Dr. Wintersteiner

in Wien. (1902) 158

Physiologie des Gesichtssinnes. Von Prof. Dr. W. A. Nagel

in Berlin. (I. Semester 1902) 428

$-\mathrm{V}$ -

Spezielle Pathologie und Therapie. "Von Dr. S chrader. in

Gera. (1902) • - 446, 519

Berieht über die ausländisehe ophthalmologische Littepatup.

Die englische Litteratur. Von Dr. Gruber in London.

(II. Semester 1902) , ... (56

Die rassische Litteratur. Von Dr. Tscherkess in Odessa, Dr. Tschemolossow in St. Petersburg

und Prof. Dr. von Ewetzky in Dorpat. (1902) ..... 234

Die französische Litteratur. Von Dr. F. Ostwalt in Paris.

(I. Semester 1902) . 308

Die polnische Litteratar. Von Dr. V. Kamocki in Warsehau.

(1902) 321

Die amerikanische Litteratur. Von Dr. K. Denig in New-York. (II. Semester 1902) 534

Sitzungsbepiehte.

Berliner ophthalmologische Gesellsehaft.

Sitzung vom 18. Juni $1903 \quad 69$

Sitzung vom 16. Juli $1903 \quad 167$

Société beige d'ophtalmologie in Brüssel.

Sitzung vom 26. April $1903 \quad 542$ 
St. I'eters burger ophthalmologische Gesellsehaft.

Sitzung vom 16. •lanuar 1903

Sitzung vom 30. •Tanuar 1903

Sitzung vom 27. Februar 1903 70

71

Sitzung vom 27. März 1903

546

Sitzung vom 17. April 1903

Gesellsehaft d er Moskauer Augenärzte.

$\mathrm{r},, \quad 30$ September .IU $\Lambda \mathrm{O}$.

Sitzung vom - - ¿i- 1903548

13. Oktober

28. Oktober ._._." $\quad \kappa .$,

bitzung vom . . $_{-}$i $-1903 \quad 549$

10. .November

Berieht über die Verhandlungev der ophthalmo-logischen Gesellsehaft zu Heidelberg. (14. bis16.

September 1903) 330

Berieht über die Verhan dlungen der $\mathrm{x} \backslash$ bteilung fürAugenlieilkunde auf der 75 .

Versammlungdeutscher Naturforscher undAerzte in Cassel1903 357

- YI

Unfall- und Vepsicherung-skunde.

Unfall- und Versicherungskunde ...... 169, 252, 466

Therapeutisehe Umschau.

Therapeutische Umschau . . . 77, 171, 254, 363. 467, 550

Offene Koppespondenz.

Oft'ene Korrespondenz

73

Tagesnachpichten und Notizen.

Tagesnachrichten und Notizen . . 79, 173, 255, 365, 470, 552

L. Littepatup - Vepzeiehnis.

Litteratur-Verzeichnis .... 79

175

255

369

470

552 